\title{
Atomic Doping Site and Dielectric Property in Perovskite Oxide Nanosheets
}

Haneul Choi ${ }^{1}$, Gwangyeob Lee ${ }^{1,3}$, Yanghee Kim ${ }^{1}$, Haena Yim ${ }^{4}$, Ji-Won Choi ${ }^{4}$, Jong Wook Roh ${ }^{5}$ and Hye Jung Chang ${ }^{1,2^{*}}$

1. Advanced Analysis Center, Korea Institute of Science and Technology, Seoul, South Korea.

2. Division of Nano \& Information Technology, KIST School, University of Science and Technology, Seoul, South Korea.

3. Department of Materials Science and Engineering, Yonsei University, Seoul, South Korea.

4. Center for Electronic Materials, Korea Institute of Science and Technology, Seoul, South Korea.

5. School of Nano \& Materials Science and Engineering, Kyungpook National University, Gyeongsangbukdo, South Korea.

* Corresponding author: almacore@kist.re.kr

Electronic devices have become miniaturized and the demand for new materials showing enhanced physical properties has dramatically increased. Two-dimensional (2D) inorganic nanosheets are one of the promising new materials. Among them, atomic-scale engineering of oxide perovskite nanosheets have been drawing keen attention due to the possibility of controlling the electronic and magnetic properties such as high- $\mathrm{T}_{\mathrm{c}}$ superconductivity, high-k ferroelectricity, and quantum magnetism [1-3].

Variety of 2D oxide nanosheets have been extensively studied recently, and thus characteristics of layered 2D nanosheets over $5 \mathrm{~nm}$ have been reported [4]. Outstanding permittivity and high dielectric strength are expected in monolayer nanosheets because nanosheet with one-unit-cell thickness is single crystal. However, the property evaluation of single nanosheets with thickness under $2 \mathrm{~nm}$ is challenging through conventional measurement method. Recently, many researchers have been studying the properties of $2 \mathrm{D}$ materials by doping various elements. The chemical substitutional doping has been used to enhance and improve the properties of materials [5]. And this method is particularly effective in modulating the electronic properties. But, the positioning of the doped elements and its effect on the properties is still unveiled. In order to confirm the doped atoms, detailed investigation on the atomic structure of 2D oxide nanosheets using (S)TEM analysis is needed.

In this study, we focused on the $2 \mathrm{D} \mathrm{Ca}_{2} \mathrm{Na}_{2} \mathrm{Nb}_{5} \mathrm{O}_{16}$ (CNNO) nanosheets fabricated by engineering the number of $\mathrm{NbO}_{6}$ octahedron and showed high-k dielectric property. By the way, high-k materials are insulating so that they are easily damaged by electron beam due to radiolysis reaction at low acceleration voltage in TEM. Besides, 2D nanosheets with large surface and consisting of low element number such as $\mathrm{O}, \mathrm{Ca}$ and $\mathrm{Na}$ are also easily damaged due to knock-on effect at high acceleration voltage. So, observation on the atomic structure of the insulating $2 \mathrm{D}$ oxide is challenging unlike conducting 2D materials such as graphene.

Unexpected damage rates under the electron beam irradiation at different acceleration voltage were observed; a damage rate was faster at low-accelerating voltage than thigh accelerating voltage. At lowacceleration voltage, a radiolysis damage mechanism was dominant than knock-on damage in the CNNO nanosheets. Based on the results, we optimized aberration corrected (S)TEM (Titan ${ }^{\text {TM }}$ 80-300, FEI) observation conditions for 2D nanosheets controlling acceleration voltage, beam current as well as sample preparation/treatment, and then directly visualized the atomic structure of the CNNO nanosheet from the TEM and STEM results as shown in Figure 1(a). Furthermore, the electrical property of one- 
unit-cell of nanosheets was measured in focused ion beam (FIB, Quanta 3D FEG, FEI) equipped with tungsten probe and $\mathrm{C}_{\mathrm{p}}$-D system (6500P, Wayne kerr). And the schematic illustration and SEM image of the connection of electrode is presented in Figure 1(b). After measuring the dielectric constant in FIB, we confirmed the thickness of the monolayer CNNO nanosheets as shown in Figure 1(c). Also, we compared the dielectric constant measured in FIB with that measured with TEM-EELS method.

\section{References:}

[1] DG Schlom et al., Journal of the American Ceramic Society 91 (2008), p. 2429.

[2] HY Hwang et al., Nature Materials 11 (2012), p. 103.

[3] J Chakhalian, AJ Millias and J Rondienlli, Nature Materials 11 (2012), p. 92.

[4] B-W Li et al., Journal of the American Chemical Society 139 (2017), p. 10868.

[5] M Osada and T Sasaki, Advanced Materials 24 (2012), p. 210.

(a)

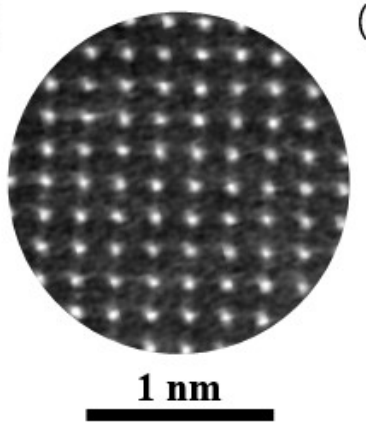

(b)

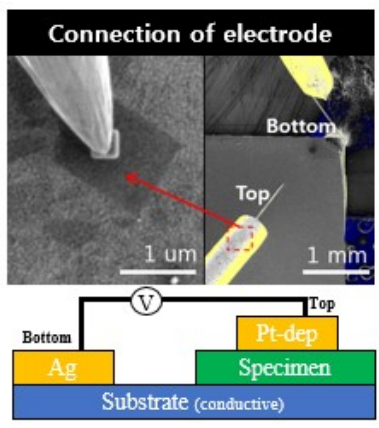

(c)

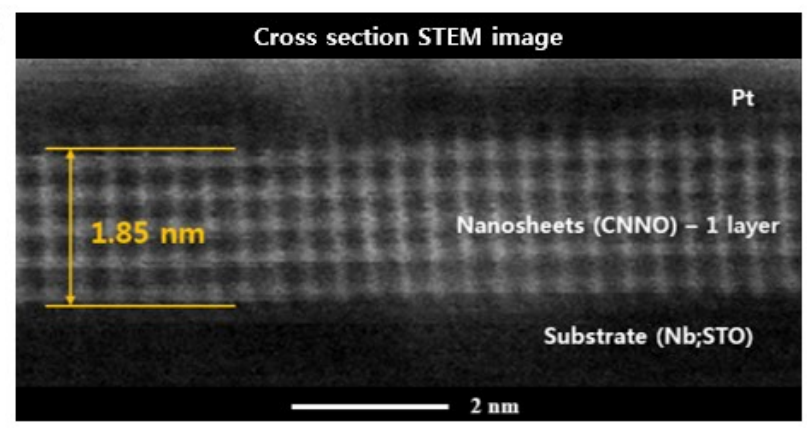

Figure 1. (a) Plane-view HRSTEM image of $\mathrm{Ca}_{2} \mathrm{Na}_{2} \mathrm{Nb}_{5} \mathrm{O}_{16}$ (CNNO) nanosheets. (b) Schematic illustration and SEM image of electrical measurement of single sheet in FIB. (c) Cross-section STEM image of monolayer CNNO nanosheet. 